\title{
Jarass, Hans D.: Charta der Grundrechte der Europäischen Union
}

\author{
504 Seiten. Verlag C. H, Beck, 2010, EUR 64. ISBN 978-3-406-60337-2
}

Es ist erfreulich, dass bereits ein knappes halbes Jahr nach dem In-Kraft-Treten der Charta der Grundrechte (GRC) der Europäischen Union per 1.12.2009 ein substanzieller Kommentar zu dieser zentralen Kodifikation aus der Feder des national und international anerkannten Staatsrechtslehrers Hans D. Jarass vorliegt. Noch erfreulicher ist es, dass sich der Autor zum Ziel gesetzt hat, im Rahmen seines Kommentars insbesondere systematische Aspekte, bedeutsame Querverweise, grundlegende dogmatische Aspekte sowie die Diskussion offener Fragestellungen in den Mittelpunkt seiner Darstellungen zu setzen und er dieses Ziel bestens erreicht, wobei gleichzeitig auf eine prägnante, gut verständliche Sprache Wert gelegt wird.

Dies lässt sich etwa am Beispiel des Art 13 GRC („Freiheit der Kunst und Wissenschaft“) treffend nachzeichnen: Nach einem substanzierten Hinweis auf die maßgebliche einschlägige Literatur werden zu dieser Bestimmung auf sechs Seiten (bzw 13 Randzahlen) die wichtigsten Aspekte des
Freiheitsrechts dargestellt. Behandelt werden in der Folge ua Grundbegriffe, Anwendungsbereich, Schutzbereich und Grundrechtsträger sowie ein Abriss zu den maßgeblichen Grundrechtseinschränkungen bzw der Rechtfertigung derselben.

Nicht nur bei der Darstellung des Art 13 GRC löst der Autor sein im Vorwort gegebenes Versprechen bestens ein, indem zahlreiche Querverweise geboten werden und letztlich sowohl juristische Laien als auch Expert/innen den Kommentar mit Gewinn lesen werden können.

Es darf dem verdienstvollen Autor sehr herzlich dazu gratuliert werden, dass er auf Anhieb einen wirklichen Standard zur Gesamtdarstellung der Charta der Grundrechte der Europäischen Union geschaffen hat, welcher sowohl bei der Rechtsanwendung als auch bei der Rechtsfortbildung eine entscheidende Rolle spielen wird.

Werner Hauser 